Review Article

\title{
Application of Machine Learning in Supply Chain Management: A Comprehensive Overview of the Main Areas
}

\author{
Erfan Babaee Tirkolaee ${ }^{D},{ }^{1}$ Saeid Sadeghi, ${ }^{2}$ Farzaneh Mansoori Mooseloo, ${ }^{3}$ \\ Hadi Rezaei Vandchali, ${ }^{4}$ and Samira Aeini ${ }^{5}$ \\ ${ }^{1}$ Department of Industrial Engineering, Istinye University, Istanbul, Turkey \\ ${ }^{2}$ Department of Industrial Management, University of Tehran, Tehran, Iran \\ ${ }^{3}$ Department of Industrial Management, University of Hormozgan, Bandar-Abbas, Iran \\ ${ }^{4}$ Australian Maritime College, University of Tasmania, Launceston, Australia \\ ${ }^{5}$ Department of Project and Construction Management, Noore Touba University, Tehran, Iran
}

Correspondence should be addressed to Erfan Babaee Tirkolaee; erfan.babaee@istinye.edu.tr

Received 13 April 2021; Revised 12 May 2021; Accepted 5 June 2021; Published 22 June 2021

Academic Editor: Ali Ahmadian

Copyright ( $) 2021$ Erfan Babaee Tirkolaee et al. This is an open access article distributed under the Creative Commons Attribution License, which permits unrestricted use, distribution, and reproduction in any medium, provided the original work is properly cited.

\begin{abstract}
In today's complex and ever-changing world, concerns about the lack of enough data have been replaced by concerns about too much data for supply chain management (SCM). The volume of data generated from all parts of the supply chain has changed the nature of SCM analysis. By increasing the volume of data, the efficiency and effectiveness of the traditional methods have decreased. Limitations of these methods in analyzing and interpreting a large amount of data have led scholars to generate some methods that have high capability to analyze and interpret big data. Therefore, the main purpose of this paper is to identify the applications of machine learning (ML) in SCM as one of the most well-known artificial intelligence (AI) techniques. By developing a conceptual framework, this paper identifies the contributions of ML techniques in selecting and segmenting suppliers, predicting supply chain risks, and estimating demand and sales, production, inventory management, transportation and distribution, sustainable development (SD), and circular economy (CE). Finally, the implications of the study on the main limitations and challenges are discussed, and then managerial insights and future research directions are given.
\end{abstract}

\section{Introduction}

In today's competitive environment, the advent of the information technology revolution, economic globalization, and high customer expectations has caused various changes in companies' supply chain management (SCM), highlighting the importance of competition among supply chains rather than companies [1-3]. SCM is the active integration of supply chain activities from original suppliers through endusers that provides services, products, and information that maximize customer value and achieve sustainable competitive advantage [4]. In the era of big data, a large amount of interactive data are routinely created, collected, and archived in different process industries, and these data are really an important asset in process operation, control, and design.
Intelligent use of these data and the extraction of information and knowledge of them have a great potential to benefit. Explosive growth in the volume of data from various parts of SCM has forced companies to develop and implement new technologies which are able to rapidly and intelligently interpret a large amount of data $[5,6]$ as the traditional decision support system cannot face big data satisfactorily. Hence, in the big data era, supply chain professionals are seeking to handle big data to reach smart supply chains.

Artificial intelligence (AI) methods are the most appropriate methods to cope with this big data-related challenge. Machine learning (ML) techniques can be considered as the popular subdisciplines in AI which identify and extract automatically the patterns among variables using large 
datasets [7]. ML algorithms can detect unknown patterns among data, generate new insights, and direct researchers towards suitable destinations. ML techniques can be used in a different context, including manufacturing, operations, healthcare, and housing [8]. Moreover, ML is widely used in managing different areas and aspects of the supply chain. Recently, ML algorithms and their applications in managing the supply chain have gained interest among researchers. Weaknesses of traditional methods in analyzing big data have led scholars to utilize ML methods that have high capabilities to analyze and interpret large volumes of data. There are many reasons to replace traditional methods with ML techniques. First, traditional methods cannot handle nonlinear problems that are widespread in real-world supply chains. ML techniques can solve this nonlinear problem satisfactorily. Second, traditional methods are incapable of dealing with large and unstructured data that come from different areas of the supply chain, where ML techniques precisely have been developed to deal with big and unstructured data. Third, ML techniques are far stronger than traditional statistical methods in recognizing and predicting the most effective factors on supply chain performance. Thus, ML can be considered as an important tool to help companies to analyze the big data in their SCM.

Seeking new information and knowledge using big data analytics can help to predict future trends, which can lead to informed decisions in various parts of SCM, making a worthy paradigm for companies. Emerging technologies such as the Internet of things (IoT), blockchain, and advanced deep learning (ADL) techniques can equip supply chains with a self-aware mechanism in which different operating objects are connected simultaneously. SCM is really in need of such a self-adaptive smart system to manage the fluctuations in customer demand. Increasing the transparency and connectivity of the supply chain via IoT and blockchain technologies can be a good sign to face the customers' ever-changing demands. This can provide a stream of real-time data and information between various parts of supply chains anywhere in the world.

There is a strong need in the literature to investigate various applications of ML techniques in different parts of supply chains as the majority of the works have considered one, two, or limited areas of the supply chain. For instance, Piramuthu [9] applied ML techniques to design an automated SCM framework. Cavalcante et al. [10] proposed a supervised ML technique for resilient supplier selection. Zhu et al. [11] used China's SME to implement ML techniques in predicting financial risks in supply chains. Priore et al. [12] applied ML techniques to find appropriate replenishment policies in SCM. Baryannis et al. [13] employed ML to predict supply chain risks. Darvazeh et al. [5] conducted a review on ML in SCM. Bai et al. [14] used a multicriteria decision-making (MCDM) and ML hybrid approach to segment suppliers based on environmental factors. However, there is less attention to accomplish a comprehensive study to consider ML applications in different related aspects of the supply chain, which can affect the understanding of how these valuable techniques can be effectively used in managing different aspects of SCM. Therefore, this paper develops a framework in which the most commonly used ML algorithms in managing different areas of the supply chain will be discussed. The main contributions of the paper are outlined as follows:

(i) By comparing the efficiency of traditional and AI methods in facing big data

(ii) By reviewing, summarizing, and classifying the most frequently used AI methods in SCM

(iii) By providing a detailed framework to explain the outputs of the application of ML techniques in supplier selection and segmentation, predicting supply chain risks, and estimating demand and sales, production, inventory management, transportation and distribution, sustainable development (SD), and circular economy (CE)

In this regard, Section 2illustrates a review of ML techniques. The applications of ML techniques in different parts of supply chain are presented in Section 3. Finally, conclusions, managerial insights, and future directions are presented in Section 4.

\section{Machine Learning}

ML techniques are used to teach machines how to automatically handle a large volume of data more efficiently. Sometimes the extraction of patterns or information from the large volume of data and interpreting them are impossible by traditional techniques [15]. The abundance of datasets available has caused a rising demand for ML techniques. ML techniques are widely applied in different industries from medicine to the military to discover and extract knowledge and information from the data. Many studies have been done by mathematicians and programmers that resulted in the development of various ML algorithms [16]. In this paper, a review of the application of machine learning in SCM was considered. Several studies have mentioned advantages of application of ML techniques in demand and sales estimation [17-19], transportation and distribution [20-22], production [23-25], inventory control $[22,26]$, supplier selection and segmentation [27-30], and so on. Some of the most famous learning algorithms with a brief description are shown in Table 1 .

\section{Machine Learning in Supply Chain Management}

Despite a large number of publications in the field of ML and SCM separately, the number of publications that have paid to the applications of ML algorithms in managing a supply chain is not adequate [38]. On the other hand, there is no enough connection between researchers and practitioners in this field. It might be caused by inadequate knowledge of practitioners about the power and advantages of ML algorithms in solving SCM problems. In this section, a review of the application of the most famous ML algorithms in managing supply chain-related issues including supplier selection, supplier segmentation, supply chain risk 
TABLE 1: Frequently used ML algorithms.

\begin{tabular}{|c|c|c|}
\hline Type of learning & Algorithm & Description \\
\hline \multirow{7}{*}{ Supervised learning } & Decision tree & $\begin{array}{c}\text { Using related values, decision trees (DTs) will categorize attributes in different groups } \\
\text { which can be applied for classification purposes [31]. }\end{array}$ \\
\hline & Naïve Bayes & Naïve Bayes can be best applied to cluster and classify objects [32]. \\
\hline & Support vector machine & $\begin{array}{c}\text { Working on the margin calculations, support vector machine (SVM) can be best } \\
\text { applied for classification purposes [33]. }\end{array}$ \\
\hline & & In $K$-nearest neighbor (KNN), the learner usually uses the training data. When the test \\
\hline & $K$-nearest neighbor & data are introduced to the learner, it compares both the data. Here, $K$ most correlated \\
\hline & & $\begin{array}{l}\text { data are taken from a training set. The majority of } K \text { is taken which serves as the new } \\
\text { class for the test data [34]. }\end{array}$ \\
\hline & Supervised neural network & $\begin{array}{l}\text { Using supervised neural network (SNN), the predicted output and actual output will } \\
\text { be compared, and according to the identified error, the parameters will be modified } \\
\text { and considered as the input into the neural network again [15]. }\end{array}$ \\
\hline \multirow{5}{*}{$\begin{array}{l}\text { Unsupervised } \\
\text { learning }\end{array}$} & $K$-means clustering & Using similarity of the clusters of data, $K$-means $(\mathrm{KM})$ clustering algorithm defines $K$ \\
\hline & & Principal component analysis (PCA) can provide faster and easier computations as it \\
\hline & analysis & reduces the dimension of the data [34]. \\
\hline & Unsunervised neural & Unsupervised neural network (UNN) categorizes data based on their similarities. \\
\hline & $\begin{array}{l}\text { Unsupervised neural } \\
\text { network }\end{array}$ & $\begin{array}{l}\text { Since the output is unknown, UNN considers the correlations between different } \\
\text { inputs and categorizes them into different groups [15]. }\end{array}$ \\
\hline \multirow[b]{2}{*}{$\begin{array}{l}\text { Semisupervised } \\
\text { learning }\end{array}$} & Self-training & $\begin{array}{c}\text { Self-training first classifies using labeled data, and then unlabeled data are used as } \\
\text { inputs [15]. }\end{array}$ \\
\hline & $\begin{array}{l}\text { Transductive support vector } \\
\text { machine }\end{array}$ & $\begin{array}{l}\text { Being an extension of SVM, transductive support vector machine (TSVM) considers } \\
\text { both labeled and unlabeled data to make sure the margin is maximized between them } \\
{[15] \text {. }}\end{array}$ \\
\hline \multirow[t]{2}{*}{ Ensemble learning } & Boosting & $\begin{array}{l}\text { Boosting uses two sorts of variables, namely, weak learners and strong learners. By } \\
\text { grouping weak learners and converting them to strong learners, it aims to decrease } \\
\text { bias and variances [36]. }\end{array}$ \\
\hline & Bagging & $\begin{array}{l}\text { Bagging is another tool which can be applied to decrease variances and increase the } \\
\qquad \text { accuracy and stability of ML [37]. }\end{array}$ \\
\hline
\end{tabular}

prediction, demand and sales estimation, production, inventory management, and transportation is provided.

\subsection{Application of Machine Learning Algorithms in Supplier} Selection. Supplier selection can be considered as the main activity in the purchasing function [39]. Due to the important role of suppliers on time, cost, and quality, supply chain managers have put much effort into the supplier selection process. The selection process can be covered by MCDM techniques which include different conflicting factors. Therefore, finding the right balance between those factors is an important task for purchasing managers. MCDM techniques support the decision-makers in evaluating a set of alternatives [27]. The MCDM techniques support decision-makers in evaluating and selecting a set of alternatives. In some cases, the number of potential suppliers and the number of criteria are significantly larger than those that MCDM techniques can cope with them satisfactorily. On the other hand, MCDM methods are categorized into descriptive and static methods like most of the other traditional methods, while in today's competitive marketplace, predictive analytics methods are definitely more useful than descriptive methods. In such an era, ML algorithms have much better performance than the mentioned methods. Among the ML techniques, DT and SVM technique as a supervised learning technique and the $\mathrm{Q}$-learning technique as a reinforcement learning (RL) technique have been applied many times by researchers to solve the supplier selection problem. In the following, how to apply some of the ML techniques to solve the supplier selection problem is discussed.

\subsubsection{Supplier Selection Using DT and P-SVM Combined} Technique. DT is one of the ML techniques that is used in classification problems where the dataset is not large and can be modeled by a similar model. Moreover, this classifier can be used when only a few features are available or a model is needed that can be visualized and explained in simpler. DT provides an effective and rapid method of classifying datasets. Recently, DT has been applied in various domains [40-42]. In addition, DTs can be used as alternatives to find solutions for a multiclass problem that has binary predictors as it has a hierarchical structure [27]. The hierarchical structure includes ramifications and nodes.

Baryannis et al. [13] compared the outputs of SVM and DT techniques in the supply chain area. The results of their study showed that SVM prediction models analyze data for classification and regression analysis. Considering the lower result for different metrics, SVM models achieve good results across various metrics. The results also indicate how accuracy can be a misleading metric in different scenarios. In the case of DT results, it can be seen that the outcomes were quite similar when it was solved via SVM. 
In this subsection, the potential support vector machine (P-SVM) is introduced as a novel approach to the original SVM proposed by Hochreiter and Obermayer [43], which is combined with DT to solve the supplier selection problem. P-SVM can be used to construct binary classifiers and select various features at the same time. The hybrid technique developed by Guo et al. [27] (based on P-SVM and DT) evaluates and selects a supplier based on three steps:

Step 1. Using training samples, it generates DT via the adapted Kruskal algorithm (for more information about the Kruskal algorithm, refer to Guo, Yuan, Tian [27], page 6984)

Step 2. P-SVM is used to construct the binary classifiers and select the features of related nodes

Step 3. Using the values of new samples as input to binary classifiers, P-SVM evaluates the suppliers and selects the best one based on DT

In the procedure of DT and P-SVM combined techniques as shown in Figure 1, at the nodes of A, B, and C, the binary classifiers are defined using the selected features. For instance, class 2 samples are separated from classes 1, 3, and 4 samples which are classified at nodes B and C. Therefore, new samples only should be defined at each of the nodes as a subset of the initial features. Moreover, to form a hierarchical system, some subsets are achieved using P-SVM. For instance, class 2 samples should be defined with a subset signed with A when they are separated from classes 1, 3, and 4 samples. Furthermore, the samples from class 3 should be described with a subset of features marked with $B$ when they are separated from samples from classes 1 and 4 . Thus, at each of the related nodes, those subsets signed with $A, B$, and $C$ shape a hierarchical system that can be used to classify samples [27].

As mentioned, the supplier selection problem is an MCDM problem in which suppliers must be evaluated with respect to many criteria. The number of criteria makes it hard for decision-makers to accomplish consistent compression in order to determine the weights of criteria and then makes it too complex to evaluate the performance of each potential supplier with respect to each weighted criteria by the traditional method. The P-SVM-DT combined technique leads to much better results than traditional MCDM and original SVM methods in solving the supplier selection problem because of the three following reasons [27]:

(I) Introducing the hierarchical structure of the P-SVM-DT combined technique reduces the number of binary classifiers

(II) Combined with the Kruskal algorithm, the hierarchical structure of P-SVM-DT is designed to increase the accuracy and decrease the computation time in different classifications

(III) To promote the generalization performance, P-SVM selects the most "informative" features to develop classifiers

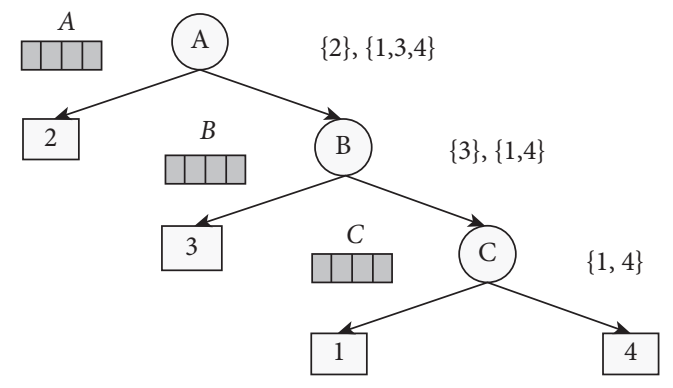

$\{j, k\} \quad$ Set of classes

\begin{tabular}{l|l|l}
\hline & $\square$
\end{tabular} Selected features at a node

$m$ Class $m$ represented as leave

FIgURE 1: Structure of P-SVM and DT combined technique [27].

3.1.2. Supplier Selection Using Reinforcement Learning. $\mathrm{RL}$, in contrast to other ML methods, does not need a model of the environment and is capable of online learning. In this subsection, Q-learning technique as one of the most widely pursued RL techniques is introduced to solve the supplier selection problem. Valluri and Croson [28] have proposed a modified Q-learning technique to evaluate and select suppliers. Q-learning is a model-free RL algorithm that rewards actions with positive outcomes and penalizes actions with negative outcomes. In this regard, $Q_{t}\left(a_{i}\right)$ indicates the supplier's value at a quality level $a_{i}$, at time $t$. Supplier's value at a quality level $a_{i}$, at the time $t+1$, is updated with the following equation:

$$
Q_{t+1}\left(a_{i}\right)=Q_{t}\left(a_{i}\right)+\alpha\left[P_{t+1}\left(a_{i}\right)+Q_{t}\left(a_{i}\right)\right]
$$

The Q-learning equation is illustrated by equation (1) where the assumption of discount factor is 1 and between periods is assumed to be 1 . Here, $P_{t+1}\left(a_{i}\right)$ is the suppliers' profits at the quality level of $a_{i}$. Various states of Markov decision process include different levels of quality which suppliers can achieve. Considering the increment of 0.1 , suppliers can produce the level of quality level ranging from 0 to 1 . The modified Q-learning algorithms have been used to obtain faster convergence. In the initial algorithm, if supplier agents meet the requirement to reach "Grade I goods," the level of quality $a_{i}$ will be selected to be rewarded; however, in the modified algorithm, all levels of quality higher than the chosen level will be rewarded and the ones lower than the chosen level of quality will be penalized (as in Figure 2(a), the 0.5 is the specific level of quality and all other levels higher than 0.5 will be rewarded). This is illustrated in Figure 2(b), where 0.5 is the chosen level of quality, and it can be seen that all other levels of quality lower than 0.5 are penalized.

The logic behind using penalty or reward actions is related to the supplier's ability to meet the requirements defined at the chosen level of quality. This means that if suppliers reach the chosen level of quality, it is highly likely 


\begin{tabular}{|l|l|l|l|l|l|l|l|l|l|l|}
\hline 0 & 0.1 & 0.2 & 0.3 & 0.4 & 0.5 & 0.6 & 0.7 & 0.8 & 0.9 & 1 \\
\hline & & & & & $X$ & & & & & \\
\hline
\end{tabular}

(a)

\begin{tabular}{|l|l|l|l|l|l|l|l|l|l|l|}
\hline 0 & 0.1 & 0.2 & 0.3 & 0.4 & 0.5 & 0.6 & 0.7 & 0.8 & 0.9 & 1 \\
\hline & & & & & $X$ & & & & & \\
\hline
\end{tabular}

(b)

FIGURE 2: RL under the modified learning algorithm [28].

to reach a higher level in the next periods, which can be rewarded by more profits. However, if suppliers fail to satisfy the requirement at the chosen level of quality, it is highly possible that the suppliers fail in the lower level of quality.

\subsection{Application of Machine Learning Algorithms in Supplier} Segmentation. Supplier segmentation is one of the strategic activities of any organization through which suppliers are categorized into groups based on their similarities. Contracts, managing, and handling suppliers' affairs are similar within each group and different from the suppliers in other groups. Supplier segmentation promotes the efficiency and effectiveness of relationships with suppliers and leads to the development and improvement of the organization's performance $[29,44]$. A review of the literature on segmentation shows that supplier segmentation has received little attention in comparison to customer segmentation and is still in its initial stages. While customer segmentation is one of the targets of the market demand side, supplier segmentation focuses on the objectives of the market supply side [30].

Generally, market segmentation can be divided into three categories as follows:

(a) Segmentation of customers or end consumers

(b) Segmentation of industrial customers or demand side of B2B2 market

(c) Segmentation of suppliers or supplied side of B2B market

While the research literature is relatively rich for the first two cases, the third case-supplier segmentation-has rather been neglected, and few studies have been conducted in order to provide a practical tool that can be applied and implemented in real-world conditions [45]. A review on supplier segmentation indicates that in most of the research studies in this area, MCDM methods under fuzzy conditions have been used to evaluate and segment the suppliers. Some of these studies are presented in Table 2.

Most of the MCDM methods that are mentioned in Table 2are expert-based and/or pairwise comparison-based methods. An important disadvantage of these methods is that the weaknesses in dealing with big data. Therefore, researchers have drawn attention to the use of ML methods in evaluating and managing suppliers [51-53] which are even more efficient than traditional methods. In this section, the application of ML in supplier segmentation has been considered. Bai et al. [14] proposed an MCDM-ML method to evaluate and segment the suppliers. In the following, the steps of MCDM-ML proposed to evaluate and segment the suppliers are presented:

Step 1. Identify a set of criteria to evaluate suppliers

Step 2. Evaluate and score the suppliers with respect to each of the weighted criteria using an outranking MCDM method (e.g., Entropy, PROMETHEE, and TOPSIS (Technique for order of preference by similarity to ideal solution (TOPSIS)))

Step 3. Determine the importance/weight of each criterion using a weighted MCDM method (e.g., Entropy, AHP, and BWM)

Step 4. Segment the suppliers based on their score using a supervised/unsupervised ML method (e.g., K-means, fuzzy $C$-means, SVM, and DT)

3.3. Application of Machine Learning Algorithms in Managing Supply Chain Risks. Supply chain risk management (SCRM) refers to the coordinated and collaborative efforts of all parties involved in a supply chain to assess, identify, monitor, and mitigate risks aiming at increasing robustness and resilience, reducing the vulnerability of the supply chain, and guaranteeing continuity and profitability [54, 55]. SCRM encompasses a wide range of actions and decisions that have resulted in an equally wide spectrum of solutions pursued by many scholars.

(1) Multiplecriteria Decision Analysis (MCDA). This category encompasses well-established approaches to assess various risk-related criteria that affect supply chain performance, as well as the efficacy of potential solutions, such as a hybrid AHP, PROMETHEE approach [56], and an integrated fuzzy TOPSIS-CRITIC approach [57, 58].

(2) Mathematical Modeling and Optimization. This category is by far the most common one and encompasses approaches based on fuzzy programming or stochastic and robust optimization $[51,54,59,60]$.

(3) AI techniques have received relatively little attention in the field of SCRM or typically supply chain research [61]. Recently, because of the availability of large amounts of data and increased computing 
TABLE 2: The widely used methods in supplier segmentation.

\begin{tabular}{lc}
\hline Authors & Method used for supplier segmentation \\
\hline $\begin{array}{l}\text { Rezaei and Ortt [46] } \\
\text { Rezaei and Ortt [45] }\end{array}$ & $\begin{array}{c}\text { Supplier segmentation using two general criteria of suppliers' capability and willingness using fuzzy logic } \\
\text { Supplier segmentation using two general criteria of suppliers' capability and willingness using fuzzy AHP }\end{array}$ \\
Rezaei et al. [47] & $\begin{array}{r}\text { Supplier segmentation using two general criteria of suppliers' capability and willingness, using BWM(Best- } \\
\text { worst method (BWM)) technique }\end{array}$ \\
Segura and Maroto [48] & $\begin{array}{r}\text { Supplier segmentation using two strategic and vital dimensions using MAUT (Multi-attribute utility theory } \\
\text { (MAUT)) and PROMETHEE (Preference ranking organization method for enrichment of evaluations } \\
\text { (PROMETHEE) techniques }\end{array}$ \\
Rezaei et al. [44] & $\begin{array}{r}\text { Supplier segmentation based on environmental criteria using ELECTRE (Elimination and choice translating } \\
\text { reality (ELECTRE)) TRI-rC techniques }\end{array}$ \\
Rezaei and Fallah lajimi [49] & $\begin{array}{r}\text { Supplier segmentation based on the hybrid approach of PPM (Purchasing portfolio matrix (PPM)) and SPP } \\
\text { (Supplier potential matrix (SPM)) matrices using BWM technique }\end{array}$ \\
Parkouhi et al. [50] & $\begin{array}{c}\text { Selection and segmentation of suppliers based on their resilience using DEMATEL (Decision-making trial } \\
\text { and evaluation laboratory (DEMATEL)) and gray SAW (Simple additive weighting (SAW)) techniques }\end{array}$ \\
\hline
\end{tabular}

power, as well as the success of ML techniques, AI has been a resurgence. It has also led to apply the potential of AI techniques in SCRM by researchers in processes such as prediction, risk identification, assessment, and response [62-69].

A data-driven framework for managing supply chain risks includes two general phases:

(i) Risk Identification. Risk identification is the first activity in SCRM. Different researchers have used different algorithms and methods to identify risks. Classification and clustering techniques such as SVM and DT are widely employed techniques to identify risks. After identifying SCR, it is the time to assess the risks and propose some appropriate activities to mitigate the risks.

(ii) Risk Assessment and Response. ML and big data analytics have also been utilized to deal with risk assessment. Artificial neural networks (ANNs) and Bayesian networks are the two most used ML techniques that have shown acceptable potential in modeling for risk assessment $[70,71]$.

In the following, a framework to integrate AI techniques within the SCRM process has been proposed that can enhance supply chain risk prediction. The aim of this two-way framework is the creation of synergy and interactivity between AI and supply chain experts: any decision that should be made by the AI experts always depends on the specific inputs by the supply chain, while the developed models and produced results have to be interpretable so that decisionmaking is based on them or they can affect SCRM decisionmaking. Figure 3represents the flow of the framework's process. On the left-hand side of the figure, the focal point is on the major tasks involved in a data-driven AI methodology. The right-hand side contains the traditional tasks that are involved in a standard SCRM process. As can be perceived, this framework is built upon effective synergies between a team of experts of data-driven AI and a team of experts of risk management within a supply chain.

3.4. Application of Machine Learning Algorithms in Demand/ Sales Estimation. In SCM, planning is based on sales/ demand estimation. By introducing nonlinear analyses, ML techniques have been used to accurately predict and forecast demand, sales, and inventory, leading to optimization of supply chain performance. An intelligent forecasting system leads to optimize performance, reduce costs, and increase sales and profit. In this case, it is required to relate the parameters associated with multiple explanatory variables to their dependent variables in a highly nonlinear manner. Being different from those traditional methods like exponential smoothing, moving average, time-series method, and Box-Jenkins method, ML techniques usually do not heavily rely on the accuracy of historical data so that ML techniques have been promoted as great alternatives for demand forecasting and planning in SCM [72]. For instance, Thomassey [73] proposed a forecasting model by advanced methods such as data mining and fuzzy logic for sale. Their model performance is more reliable than traditional models. This model worked well under situations such as strong seasonality of sales, volatile demand, and a wide number of items with a short life cycle or the lack of historical data background. Figure 4indicates the principles of a fuzzy forecasting system. This system makes it possible to generate a model which automatically recreates the expert approach when they carry out intuitive predictions with respect to explanatory variables. Three stages are needed to implement this fuzzy forecasting system as follows:

(1) Removing the impact of explanatory variables from historical sales

(2) Using the baseline sales as a resulting data to forecast the baseline of the next year, for example, applying a basic forecasting technique according to the seasonality average

(3) Adding the impact of explanatory variables regarding the next year to this baseline forecast

3.5. Application of Machine Learning Algorithms in Inventory Management. Storage incurs important costs in SCM. For instance, the annual costs of storage in supply chains are about $15 \%-35 \%$ of their total business value [74]. Supply chain inventory management seeks to decrease costs, increase product variety, and improve customer service as well. However, precisely estimating, predicting, and 


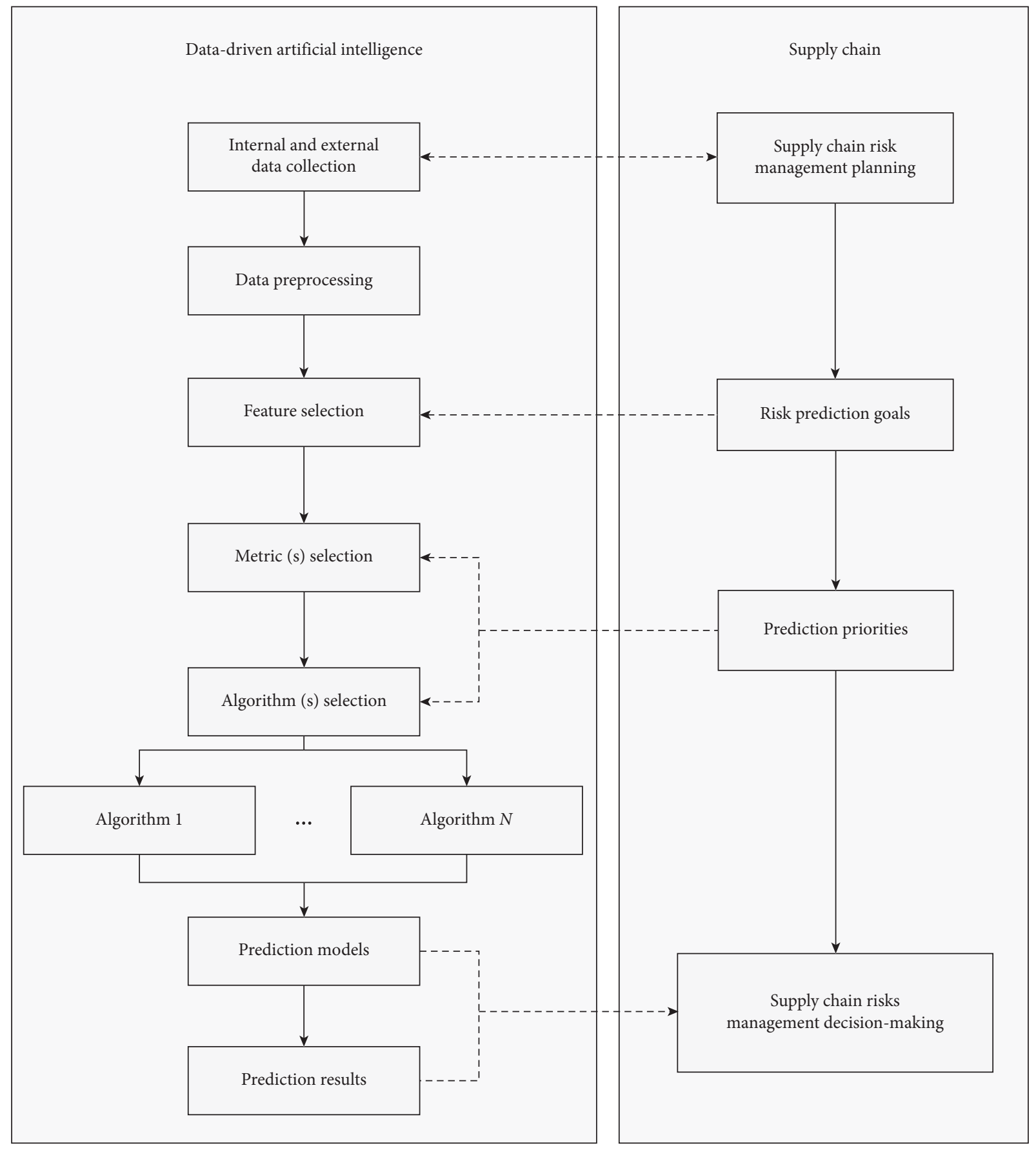

FigURE 3: Data-driven risk prediction framework [54].

accessing the concerned information of all these goals by traditional decision rules are hard because this information usually is high according to the experience and judgment of inventory managers themselves. Hence, the inventory input is an uncertain parameter. In recent years, the inefficiency of traditional methods in facing uncertainty has led researchers to apply AI. ML tools can explore quick input comparable patterns on warehouse datasets. Gumus et al. [26] applied neural networks to lead-time forecasting. They employed neuro-fuzzy demand in a multi-echelon supply chain. The results showed that their proposed model efficiently improved the inventory management performance. Furthermore, ML has demonstrated an efficient performance for in-house delivery or automating inspecting the damage inside logistics hubs. As a result, the ML tools are able to find the hidden inventory patterns that have never been unfolded in decreasing and saving costs. 


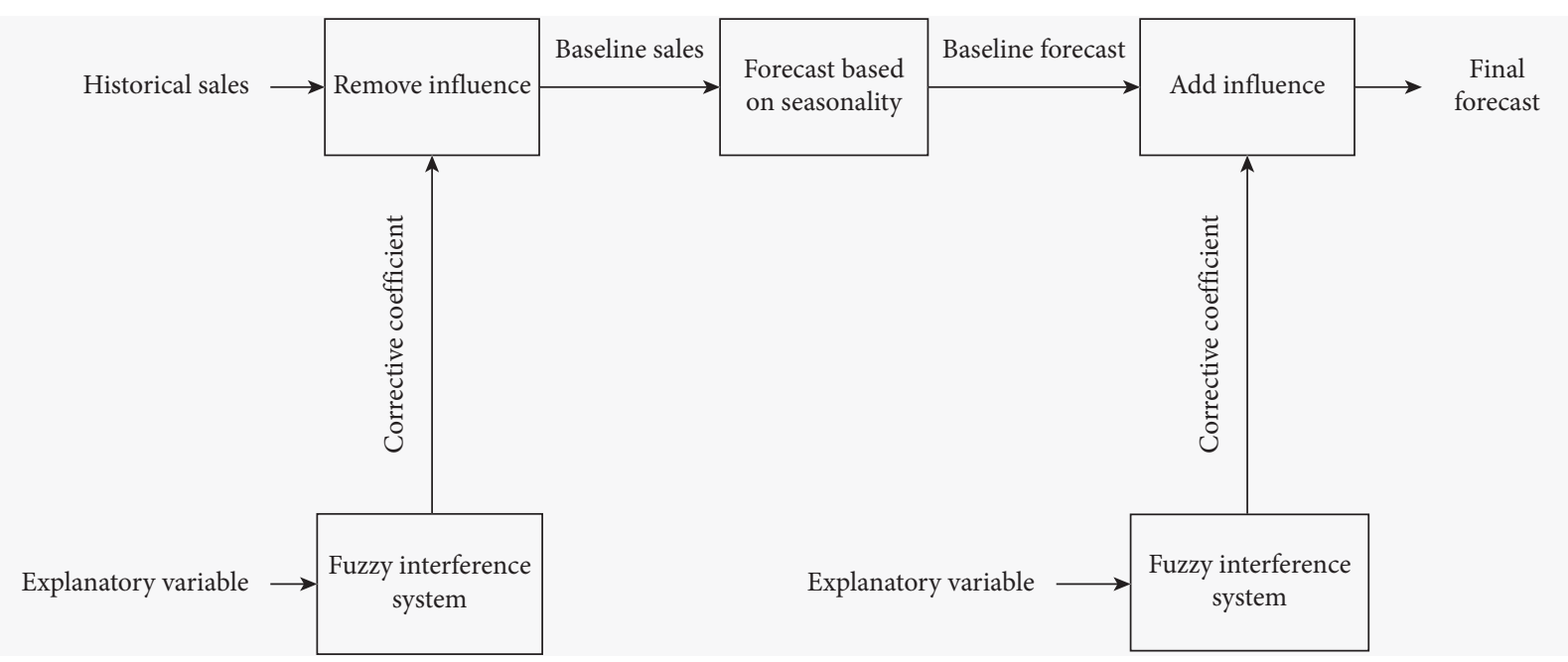

Figure 4: Fuzzy forecasting system [73].

3.6. Application of Machine Learning Algorithms in Transportation and Distribution. Vehicle routing problems (VRPs) are categorized as one of the most applicable issues in SCM [75-80]. Solving VRPs is one of the high and wellknown applications of ML in SCM. The optimal route for transporting vehicles is an important issue in SCM to deliver products and services on time to customers; in most cases, routing is beyond the ability of a human brain. ML tools have excellent performance for analyzing large and diverse datasets and accurate forecasting of parameters. Ćirović et al. [81] developed a model for the routing of light delivery vehicles through logistics operators in which an adaptive neural network was trained by a simulated annealing algorithm. This model assesses the performance of the distribution network routes. ML algorithms are able to generate better delivery routes by objectively and timely exploring the pattern of transportation, consumers' behavior, infrastructures, and vehicles.

3.7. Application of Machine Learning Algorithms in Production. ML tools will improve the production planning and factory scheduling accuracy by taking into account multiple constraints. ML tools will also make it possible to balance the constraints more effectively than those that were manually done in the past, particularly for manufacturers who rely on build-to-order and make-to-stock production workflows. Manufacturers can apply ML tools to reduce supply chain latency for components and parts used in their most heavily customized products. For example, faced with the different production regulations and customization requirements of each country, Chen et al. [82] applied NNs to group similar customization needs. Then, they used the existing inventory information to select the parts for production managers, which hugely reduced the cost during the supply chain compared with human decision. As a whole, the ML tools can yield the lead-time prediction in production with a shorter response time.
3.8. Application of Machine Learning Algorithms in Sustainable Development. In different industries, the emergence of decentralization in the level of organizational structure restricts the SCM scope whereas concentrating on sustainable performance [83-87]. In terms of quantity and time, ML has real-time capability inputs related to activities and resources [88]. Accordingly, ML gathers and employs the data, conducts analysis, and renders the required inputs to decide on coping with the dealt affairs. ML permits industries to minimize human intervention and maximize the effectiveness and efficiency of work processes simultaneously.

Focusing on customer demand and production planning in traditional SCM is not an effective way, and that is why the concept of sustainable SCM is brought up [89]. Furthermore, the lack of objectivity in human experts limits the research works on the data analysis on demand forecast in the traditional SCM. In fact, the SCM literature shows that analysis in the traditional supply chain is highly affected by the biases of decision-makers and unreasonable market conjectures. Due to this, the inclusion of ML is the best choice. For example, ML provides better production planning since it helps managers identify consumers' requirements and their purchase patterns under different scenarios. On the other hand, for a sustainable SCM, ML can play a vital role due to its high potential to tackle the uncertainty and conserve industries for efficiently encountering the approaching challenges. As a real case, Sharma et al. [89] performed a comprehensive review on the application of ML techniques in sustainable agriculture SCM, where 93 papers were surveyed.

\subsection{Application of Machine Learning Algorithms in Circular} Economy. A CE is a systemic procedure that contributes to maintain the activities related to reverse flows in supply chains including resource usage, recovery, recycling, reuse, repair, remanufacturing, and refurbishment [90]. It motivates the perpetual reuse of materials to reduce waste, as well as directing demand toward natural resource consumption 


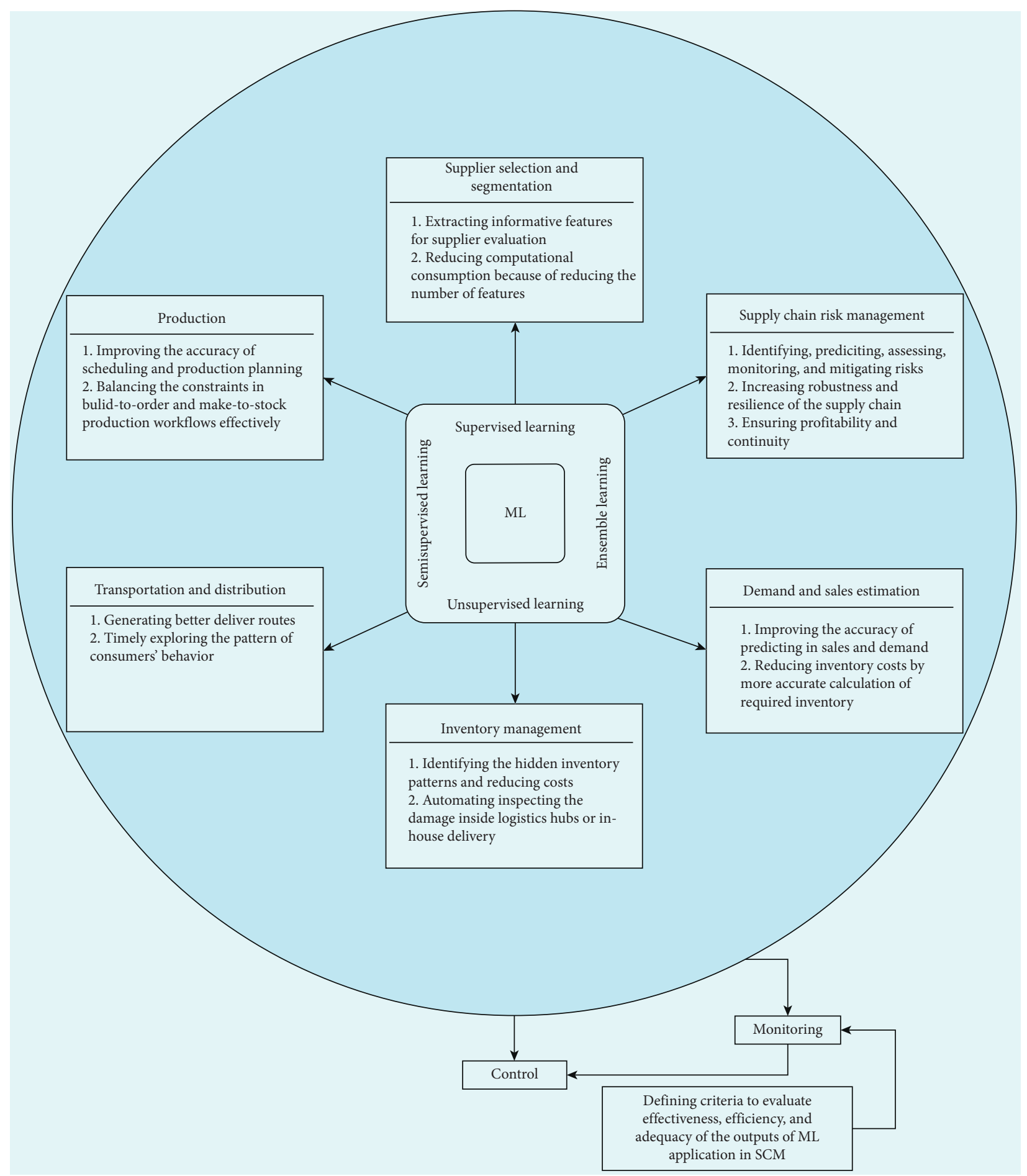

FIGURE 5: Conceptual framework for ML application in SCM.

[86]. AI and ML algorithms can serve as efficient platforms for a quick transformation to the CE. There are three key factors of CE where ML techniques can be applied as follows: (i) Design Circular Materials, Components, and Products. It is obvious that ML tools can facilitate the development of new materials and products, such as rapid ML-driven prototyping and testing 
(ii) Operate Circular Business Models. ML can improve product circulation through intelligent demand prediction, pricing, inventory management, predictive maintenance, and so on

(iii) Optimize Circular Infrastructure. ML can enhance components remanufacturing, products sorting and disassembling, and materials recycling which can provide the reverse logistics infrastructure

As a recent study, Wilts et al. [91] investigated the application of AI in sorting of municipal solid waste (MSW) according to the CE principles. ML was discussed as one of the most efficient technologies for automatic waste sorting.

\section{A Framework for ML Application in SCM}

In the previous sections, the application of ML techniques in different areas of SCM was discussed. In this section, a conceptual framework was presented to give a broad overview of the outputs of the application of ML in each area of SCM, which cannot be achieved by applying traditional methods. Moreover, some useful information about the necessary and sufficient conditions to use ML in SCM effectively was provided. As it is clear in Figure 5, one of the most important outputs of applying ML techniques such as SVM in supplier evaluation is to extract some seminal features which could have a significant effect on supplier selection and segmentation processes. Moreover, using ML techniques can reduce the time of evaluating suppliers based on criteria by reducing the number of features applied to classification. The outputs of applying ML in SCRM are predicting, assessing, and mitigating risks intelligently. Furthermore, applying ML in SCRM can make it possible to cultivate a robust and resilient supply chain and ensure the continuity and profitability of the organization. One of the outputs of applying ML in demand and sales estimation is to predict demand and sales more accurately than traditional methods, and this leads to lower inventory costs by an accurate required inventory estimation. ML techniques can recognize the hidden inventory patterns that have a significant impact on reducing costs. Using AI makes it possible to establish an automated inspecting system to mitigate the damage inside logistics hubs or in-house delivery. Transportation and distribution is an important activity in SCM that loads heavy costs on organizations. The results of applying ML in transportation and distribution systems can lead to timely delivery of a product to the corresponding customers by generating better delivery routes and exploring the consumer behavior. The other activity in SCM that has faced multiple constraints is production planning. An appropriate production planning system should be dealt with these constraints effectively. ML techniques can balance the constraints in build-toorder and make-to-stock production workflows and effectively improve the accuracy of scheduling and production planning.

As mentioned earlier, traditional methods are weak in dealing with big data problems. Therefore, recently, researchers have drawn attention to the use of ML techniques in managing the supply chain $[13,14,52,53]$ which are even more efficient than traditional techniques in dealing with a large number of data. On the other hand, there are two criticisms about ML methods as follows:

(I) The nature of most of the AI-based techniques is learning historical data, and then they make a decision about new samples. These techniques are not capable of dealing with big changes and revolutions in problem situations. For example, the COVID-19 outbreak has made large and unpredictable changes in the business environments that ML techniques could not have acceptable performance in dealing with. In such a condition that the problem situation is undergoing big and unpredictable changes, AI is faced with a condition that has no resemblance to what it has learned and faced in the past, and the effectiveness and efficiency of AI methods could be dramatically reduced.

(II) There is a doubt about the validity and fairness of ML techniques in some cases. At the heart of the problem is the fact that ML techniques calculate optimal models from the data they are given. Meaning they can end up replicating the problems they are meant to correct [92].

Therefore, as mentioned at the bottom of Figure 5, the outputs of ML techniques in SCM must be monitored, evaluated, and controlled based on their logical desirability and cultural feasibility. In other words, the outputs of AIbased methods must be evaluated by the human agent (SCM expert) to ensure that the outputs and gained strategies from them are logically desirable and culturally feasible or not, or the outputs of ML application in SCM are effective, efficient, and adequate.

\section{Conclusion and Managerial Implication and Future Research Direction}

This paper aimed to provide an overview of the application of ML techniques in different areas of the supply chain. In this regard, after a brief review of widely used supervised, unsupervised, and semisupervised ML techniques, the application of each one in managing different areas of the supply chain was presented. Accordingly, supplier selection, supplier segmentation, supply chain risk prediction, demand estimation, inventory management, transportation, sustainable development, and circular economy in SCM were discussed as the most significant areas. In the section on supplier selection, two different machine learning methods were explained. First, how the use of DT and P-SVM combined method in evaluating and selecting suppliers was explained. Second, the Q-learning technique as an RL technique was introduced in order to solve the supplier selection problem. In the next section, the procedure of supplier segmentation using an MCDM-FCM combined method and based on environmental criteria was described. Third, the stages of designing a data-driven framework for 
predicting supply chain risks were introduced. Fourth, the application of a fuzzy forecasting system as one of the widely used machine learning techniques in demand/sales estimation was explained. Finally, a summary of ML algorithms' applications in inventory management, transportation, and production was introduced. Although the main goal of this paper was addressed as expected and the advantages of applying ML in managing supply chains were concluded, there are some limitations. For instance, considering the target audience of this paper (beginners and industry managers), providing the details of ML algorithms was not possible and just general explanation and sometimes drawing some flowcharts to acquaint the beginners and managers with the process of implementing and utilizing the power of ML algorithms in the SCM area were provided. In this paper, without any concentration on a specific industry, the application of ML generally in different SCs was considered; it is obvious that the nature of the industry and the type and the volume of data have a significant effect on selecting a suitable algorithm. Therefore, it is suggested that managers should be careful in using different algorithms and consider the suitability of the selected algorithm with the nature of the data and its interpretability for the industry. There are some industries that are in the early stages of using ML techniques to improve their various supply chain processes. For example, there is a great space in the renewable energy supply chain to cover and fruitful research contents to see in this domain in the future. There is also a great gap in leveraging the power of the mathematical optimizing model and machine learning in order to design and optimize SC, which can be considered in future research studies. Furthermore, the presented framework can be tested using structural equation modeling in some industries in future research studies. Despite the presence of AI for the last half-century and its recent emergence in the SCM area, there is still a lack of research regarding the specific topics of artificial intelligence for different areas of the supply chain. Based on projected AI research trends, we suggest further exploration in the application of RL techniques in real-time pricing. Most AI techniques applications in the SCM area remain limited to relatively well-structured (well-defined), operational, and tactical SCM problems. Future research can address the application of AI techniques (especially agentbased systems) to solve a variety of soft but strategic SCM issues. The main reason is that providing efficient solutions for SCM problem is either too expensive or difficult due to the inherent complexity and ill-structured nature of the problem, such as outsourcing relationships, supplier relationship management, supply chain coordination, and strategic alliances among supply chain partners.

\section{Conflicts of Interest}

The authors declare that they have no conflicts of interest.

\section{References}

[1] Z. Dai, F. Aqlan, K. Gao, and Y. Zhou, "A two-phase method for multi-echelon location-routing problems in supply chains," Expert Systems with Applications, vol. 115, pp. 618634, 2019.

[2] S. C. Koh, S. Saad, and S. Arunachalam, "Competing in the 21st century supply chain through supply chain management and enterprise resource planning integration," andInternational Journal of Physical Distribution and Logistics Management, vol. 36, no. 6, pp. 455-465, 2006.

[3] S. Sadeghi, N. Rasouli, and G. Jandaghi, "Identifying and prioritizing contributing factors in supply chain competitiveness by using PLS-BWM techniques (case study: payam shoes company)," World Scientific News, vol. 49, no. 2, pp. 117-143, 2016.

[4] D. M. Lambert, M. C. Cooper, and J. D. Pagh, "Supply chain management: implementation issues and research opportunities," The International Journal of Logistics Management, vol. 9, no. 2, pp. 1-20, 1998.

[5] S. S. Darvazeh, I. R. Vanani, and F. M. Musolu, "Big data analytics and its applications in supply chain management," in New Trends in the Use of Artificial Intelligence for the Industry 4.0, IntechOpen, London, UK, 2020.

[6] S. Tiwari, H. M. Wee, and Y. Daryanto, "Big data analytics in supply chain management between 2010 and 2016: insights to industries," Computers and Industrial Engineering, vol. 115, pp. 319-330, 2018.

[7] B. Biggio and F. Roli, "Wild patterns: ten years after the rise of adversarial machine learning," Pattern Recognition, vol. 84, pp. 317-331, 2018.

[8] F. M. Musolu, S. Sadeghi Darvazeh, and I. Raeesi Vanani, "Deep learning and its applications in medical imaging," in Internet of Things for Healthcare Technologies, pp. 137-153, Springer, Singapore, 2021.

[9] S. Piramuthu, "Machine learning for dynamic multi-product supply chain formation," Expert Systems with Applications, vol. 29, no. 4, pp. 985-990, 2005.

[10] I. M. Cavalcante, E. M. Frazzon, F. A. Forcellini, and D. Ivanov, "A supervised machine learning approach to datadriven simulation of resilient supplier selection in digital manufacturing," International Journal of Information Management, vol. 49, pp. 86-97, 2019.

[11] Y. Zhu, C. Xie, G.-J. Wang, and X.-G. Yan, "'Predicting China's SME credit risk in supply chain finance based on machine learning methods," Entropy, vol. 18, no. 5, p. 195, 2016.

[12] P. Priore, B. Ponte, R. Rosillo, and D. de la Fuente, “Applying machine learning to the dynamic selection of replenishment policies in fast-changing supply chain environments," International Journal of Production Research, vol. 57, no. 11, pp. 3663-3677, 2019.

[13] G. Baryannis, S. Dani, and G. Antoniou, "Predicting supply chain risks using machine learning: the trade-off between performance and interpretability," Future Generation Computer Systems, vol. 101, pp. 993-1004, 2019.

[14] C. Bai, J. Rezaei, and J. Sarkis, "Multicriteria green supplier segmentation," IEEE Transactions on Engineering Management, vol. 64, no. 4, pp. 515-528, 2017.

[15] A. Dey, "Machine learning algorithms: a review," International Journal of Computer Science and Information Technologies, vol. 7, no. 3, pp. 1174-1179, 2016.

[16] M. Bowles, Machine Learning in Python: Essential Techniques for Predictive Analysis, John Wiley and Sons, Hoboken, NJ, USA, 2015.

[17] R. Carbonneau, K. Laframboise, and R. Vahidov, "Application of machine learning techniques for supply chain demand 
forecasting," European Journal of Operational Research, vol. 184, no. 3, pp. 1140-1154, 2008.

[18] A. Ning, H. Lau, Y. Zhao, and T. T. Wong, "Fulfillment of retailer demand by using the MDL-optimal neural network prediction and decision policy," IEEE Transactions on Industrial Informatics, vol. 5, no. 4, pp. 495-506, 2009.

[19] Y. Pan, R. Pavur, and T. Pohlen, "Revisiting the effects of forecasting method selection and information sharing under volatile demand in SCM applications," IEEE Transactions on Engineering Management, vol. 63, no. 4, pp. 377-389, 2016.

[20] M. Maghrebi, C. Sammut, and S. T. Waller, "Feasibility study of automatically performing the concrete delivery dispatching through machine learning techniques," Engineering, Construction and Architectural Management, vol. 22, no. 5, pp. 573-590, 2015.

[21] S. Mercier and I. Uysal, "Neural network models for predicting perishable food temperatures along the supply chain," Biosystems Engineering, vol. 171, pp. 91-100, 2018.

[22] S. Shervais, T. T. Shannon, and G. G. Lendaris, "Intelligent supply chain management using adaptive critic learning," IEEE Transactions on Systems, Man, and Cybernetics - Part A: Systems and Humans, vol. 33, no. 2, pp. 235-244, 2003.

[23] W. W. C. Chung, K. C. M. Wong, and P. T. K. Soon, "An ANN-based DSS system for quality assurance in production network," Journal of Manufacturing Technology Management, vol. 18, no. 7, pp. 836-857, 2007.

[24] C. Liu, T. Shu, S. Chen, S. Wang, K. K. Lai, and L. Gan, “An improved grey neural network model for predicting transportation disruptions," Expert Systems with Applications, vol. 45, pp. 331-340, 2016.

[25] H. Wu, G. Evans, and K.-H. Bae, "Production control in a complex production system using approximate dynamic programming," International Journal of Production Research, vol. 54, no. 8, pp. 2419-2432, 2016.

[26] A. T. Gumus, A. F. Guneri, and F. Ulengin, "A new methodology for multi-echelon inventory management in stochastic and neuro-fuzzy environments," International Journal of Production Economics, vol. 128, no. 1, pp. 248-260, 2010.

[27] X. Guo, Z. Yuan, and B. Tian, "Supplier selection based on hierarchical potential support vector machine," Expert Systems with Applications, vol. 36, no. 3, pp. 6978-6985, 2009.

[28] A. Valluri and D. Croson, "Agent learning in supplier selection models," Decision Support Systems, vol. 39, no. 2, pp. 219-240, 2005.

[29] W. Jiang and J. Liu, "Inventory financing with overconfident supplier based on supply chain contract," Mathematical Problems in Engineering, vol. 2018, Article ID 5054387, 12 pages, 2018.

[30] S. Erevelles and T. H. Stevenson, "Enhancing the business-tobusiness supply chain: insights from partitioning the supplyside," Industrial Marketing Management, vol. 35, no. 4, pp. 481-492, 2006.

[31] S. B. Kotsiantis, I. D. Zaharakis, and P. E. Pintelas, "Machine learning: a review of classification and combining techniques," Artificial Intelligence Review, vol. 26, no. 3, pp. 159-190, 2006.

[32] D. Lowd and P. Domingos, "Naive Bayes models for probability estimation," in Proceedings of the 22nd International Conference on Machine Learning, pp. 529-536, Germany, August 2005.

[33] A. Karatzoglou, D. Meyer, and K. Hornik, "Support vector machines in R," Journal of Statistical Software, vol. 15, no. 9, pp. 1-28, 2006.
[34] P. Harrington, Machine Learning in Action, Manning Publications Co, Shelter Island, NY, USA, 2012.

[35] S. Shalev-Shwartz, Y. Singer, N. Srebro, and A. Cotter, "Pegasos: primal estimated sub-gradient solver for SVM," Mathematical Programming, vol. 127, no. 1, pp. 3-30, 2011.

[36] Z.-H. Zhou, "When semi-supervised learning meets ensemble learning," in Proceedings of the International Workshop on Multiple Classifier Systems, pp. 529-538, Springer, Reykjavik, Iceland, June 2009.

[37] A. Lemmens and C. Croux, "Bagging and boosting classification trees to predict churn," Journal of Marketing Research, vol. 43, no. 2, pp. 276-286, 2006.

[38] M. Bertolini, D. Mezzogori, M. Neroni, and F. Zammori, "Machine learning for industrial applications: a comprehensive literature review," Expert Systems with Applications, vol. 175, Article ID 114820, 2021.

[39] Q. Pang, T. Yang, M. Li, and Y. Shen, "A fuzzy-grey multicriteria decision making approach for green supplier selection in low-carbon supply chain," Mathematical Problems in Engineering, vol. 2017, Article ID 9653261, 9 pages, 2017.

[40] G. A. Levow, "Characterizing and recognizing spoken corrections in human-computer dialogue," in Proceedings of the 36th Annual Meeting of the Association for Computational Linguistics and 17th International Conference on Computational Linguistics, vol. 1, pp. 736-742, Quebec, Canada, August 1998.

[41] M. K. Markey, G. D. Tourassi, and C. E. Floyd, "Decision tree classification of proteins identified by mass spectrometry of blood serum samples from people with and without lung cancer," Proteomics, vol. 3, no. 9, pp. 1678-1679, 2003.

[42] B. Zmazek, L. Todorovski, S. Džeroski, J. Vaupotič, and I. Kobal, "Application of decision trees to the analysis of soil radon data for earthquake prediction," Applied Radiation and Isotopes, vol. 58, no. 6, pp. 697-706, 2003.

[43] S. Hochreiter and K. Obermayer, "Support vector machines for dyadic data," Neural Computation, vol. 18, no. 6, pp. 1472-1510, 2006.

[44] J. Rezaei, M. Kadziński, C. Vana, and L. Tavasszy, "Embedding carbon impact assessment in multi-criteria supplier segmentation using ELECTRE TRI-rC," Annals of Operations Research, pp. 1-23, 2017.

[45] J. Rezaei and R. Ortt, "Multi-criteria supplier segmentation using a fuzzy preference relations based AHP," European Journal of Operational Research, vol. 225, no. 1, pp. 75-84, 2013.

[46] J. Rezaei and R. Ortt, "A multi-variable approach to supplier segmentation," International Journal of Production Research, vol. 50, no. 16, pp. 4593-4611, 2012.

[47] J. Rezaei, J. Wang, and L. Tavasszy, "Linking supplier development to supplier segmentation using Best Worst Method," Expert Systems with Applications, vol. 42, no. 23, pp. 9152-9164, 2015.

[48] M. Segura and C. Maroto, "A multiple criteria supplier segmentation using outranking and value function methods," Expert Systems with Applications, vol. 69, pp. 87-100, 2017.

[49] J. Rezaei and H. Fallah Lajimi, "Segmenting supplies and suppliers: bringing together the purchasing portfolio matrix and the supplier potential matrix," International Journal of Logistics Research and Applications, vol. 22, pp. 1-18, 2018.

[50] S. V. Parkouhi, A. S. Ghadikolaei, and H. F. Lajimi, "Resilient supplier selection and segmentation in grey environment," Journal of Cleaner Production, vol. 207, pp. 1123-1137, 2019.

[51] G. Baryannis, S. Dani, S. Validi, and G. Antoniou, "Decision support systems and artificial intelligence in supply chain risk 
management," in Revisiting Supply Chain Risk, pp. 53-71, Springer, Cham, Switzerland, 2019.

[52] A. Bruzzone and A. Orsoni, "AI and simulation-based techniques for the assessment of supply chain logistic performance," in Proceedings of the 36th Annual Simulation Symposium, pp. 154-164, IEEE, Florida, FL, USA, March 2003.

[53] K. Zhao and X. Yu, "A case based reasoning approach on supplier selection in petroleum enterprises," Expert Systems with Applications, vol. 38, no. 6, pp. 6839-6847, 2011.

[54] G. Baryannis, S. Validi, S. Dani, and G. Antoniou, "Supply chain risk management and artificial intelligence: state of the art and future research directions," International Journal of Production Research, vol. 57, no. 7, pp. 2179-2202, 2019.

[55] J. Luo, X. Zhang, and X. Jiang, "Multisources risk management in a supply chain under option contracts," Mathematical Problems in Engineering, vol. 2019, Article ID 7482584, 12 pages, 2019.

[56] S. Prasanna Venkatesan and S. Kumanan, "Supply chain risk prioritisation using a hybrid AHP and PROMETHEE approach," International Journal of Services and Operations Management, vol. 13, no. 1, pp. 19-41, 2012.

[57] R. Rostamzadeh, M. K. Ghorabaee, K. Govindan, A. Esmaeili et al., "Evaluation of sustainable supply chain risk management using an integrated fuzzy TOPSIS- CRITIC approach," Journal of Cleaner Production, vol. 175, pp. 651-669, 2018.

[58] M. Abdel-Basset and R. Mohamed, "A novel plithogenic TOPSIS- CRITIC model for sustainable supply chain risk management," Journal of Cleaner Production, vol. 247, Article ID 119586, 2020.

[59] Y. H. Lee, P. Golinska-Dawson, and J. Z. Wu, "Mathematical models for supply chain management," Mathematical Problems in Engineering, vol. 2016, Article ID 6167290, 4 pages, 2016.

[60] S. S. Darvazeh, M. Amiri, and F. M. Mooseloo, "Artificial intelligence and its application in data-driven optimization," in The Open Access Book, Data Mining, IntechOpen, London, UK, 2021.

[61] R. Toorajipour, V. Sohrabpour, A. Nazarpour, P. Oghazi, and M. Fischl, "Artificial intelligence in supply chain management: a systematic literature review," Journal of Business Research, vol. 122, pp. 502-517, 2021.

[62] H. Chen, Y. Zhu, K. Hu, and X. Li, "Virtual enterprise risk management using artificial intelligence," Mathematical Problems in Engineering, vol. 2010, Article ID 572404, 20 pages, 2010.

[63] Y. Fan, L. Heilig, and S. Voß, "Supply chain risk management in the era of big data," in Design, User Experience, and Usability: Design Discourse, pp. 283-294, Springer, Cham, Switzerland, 2015.

[64] M. He, H. Ji, Q. Wang, C. Ren, and R. Lougee, "Big data fueled process management of supply risks: sensing, prediction, evaluation and mitigation," in Proceedings of the Winter Simulation Conference 2014, pp. 1005-1013, IEEE, Savannah, GA, USA, December 2014.

[65] D. Zage, K. Glass, and R. Colbaugh, "Improving supply chain security using big data," in Proceedings of the 2013 IEEE International Conference on Intelligence and Security Informatics, pp. 254-259, IEEE, Seattle, WA, USA, June 2013.

[66] S. Ye, Z. Xiao, and G. Zhu, "Identification of supply chain disruptions with economic performance of firms using multicategory support vector machines," International Journal of Production Research, vol. 53, no. 10, pp. 3086-3103, 2015.
[67] W. Yan, J. He, and A. J. C. Trappey, "Risk-aware supply chain intelligence: AI-enabled supply chain and logistics management considering risk mitigation," Advanced Engineering Informatics, vol. 42, Article ID 100976, 2019.

[68] L. Malviya, P. Chittora, P. Chakrabarti, R. S. Vyas, and S. Poddar, "Backorder prediction in the supply chain using machine learning," Materials Today: Proceedings, 2021.

[69] S. S. Kamble, A. Gunasekaran, V. Kumar, A. Belhadi, and C. Foropon, "A machine learning based approach for predicting blockchain adoption in supply chain," Technological Forecasting and Social Change, vol. 163, Article ID 120465, 2021.

[70] S.-S. Leu and C.-M. Chang, "Bayesian-network-based safety risk assessment for steel construction projects," Accident Analysis and Prevention, vol. 54, pp. 122-133, 2013.

[71] L. Zhang, X. Wu, M. J. Skibniewski, J. Zhong, and Y. Lu, "Bayesian-network-based safety risk analysis in construction projects," Reliability Engineering and System Safety, vol. 131, pp. 29-39, 2014.

[72] D. Ni, Z. Xiao, and M. K. Lim, "A systematic review of the research trends of machine learning in supply chain management," International Journal of Machine Learning and Cybernetics, pp. 1-20, 2019.

[73] S. Thomassey, "Sales forecasts in clothing industry: the key success factor of the supply chain management," International Journal of Production Economics, vol. 128, no. 2, pp. 470-483, 2010.

[74] S. G. Timme and C. Williams-Timme, "The real cost of holding inventory," Supply Chain Management Review, vol. 7, no. 4, pp. 30-37, 2003.

[75] E. B. Tirkolaee, A. Goli, A. Faridnia, M. Soltani, and G.-W. Weber, "Multi-objective optimization for the reliable pollution-routing problem with cross-dock selection using Pareto-based algorithms," Journal of Cleaner Production, vol. 276, Article ID 122927, 2020.

[76] M. Alinaghian, E. B. Tirkolaee, Z. K. Dezaki, S. R. Hejazi, and W. Ding, "An augmented Tabu search algorithm for the green inventory-routing problem with time windows," Swarm and Evolutionary Computation, vol. 60, Article ID 100802, 2021.

[77] E. B. Tirkolaee and N. S. Aydın, "A sustainable medical waste collection and transportation model for pandemics," Waste Management and Research, Article ID 0734242X211000437, 2021.

[78] E. B. Tirkolaee, P. Abbasian, and G.-W. Weber, "Sustainable fuzzy multi-trip location-routing problem for medical waste management during the COVID-19 outbreak," Science of the Total Environment, vol. 756, Article ID 143607, 2021.

[79] M. Yousefi Nejad Attari, A. Ebadi Torkayesh, B. Malmir, and E. Neyshabouri Jami, "Robust possibilistic programming for joint order batching and picker routing problem in warehouse management," International Journal of Production Research, pp. 1-19, 2021.

[80] N. Furian, M. O’Sullivan, C. Walker, and E. Çela, "A machine learning-based branch and price algorithm for a sampled vehicle routing problem," OR Spectrum, pp. 1-40, 2021.

[81] G. Ćirović, D. Pamučar, and D. Božanić, "Green logistic vehicle routing problem: routing light delivery vehicles in urban areas using a neuro-fuzzy model," Expert Systems with Applications, vol. 41, no. 9, pp. 4245-4258, 2014.

[82] M.-K. Chen, T.-W. Tai, and T.-Y. Hung, "Component selection system for green supply chain," Expert Systems with Applications, vol. 39, no. 5, pp. 5687-5701, 2012.

[83] H. R. Vandchali, S. Cahoon, and S.-L. Chen, "Creating a sustainable supply chain network by adopting relationship 
management strategies," Journal of Business-To-Business Marketing, vol. 27, no. 2, pp. 125-149, 2020.

[84] H. R. Vandchali, S. Cahoon, and S.-L. Chen, "The impact of supply chain network structure on relationship management strategies: an empirical investigation of sustainability practices in retailers," Sustainable Production and Consumption, vol. 28, pp. 281-299, 2021.

[85] H. R. Vandchali, S. Cahoon, and S. L. Chen, "The impact of power on the depth of sustainability collaboration in the supply chain network for Australian food retailers," International Journal of Procurement Management, vol. 14, no. 2, pp. 165-184, 2021.

[86] D. Wang and Y. Zhang, "Implications for sustainability in supply chain management and the circular economy using machine learning model," Information Systems and E-Business Management, pp. 1-13, 2020.

[87] P. Wang, "On defining artificial intelligence," Journal of Artificial General Intelligence, vol. 10, no. 2, pp. 1-37, 2019.

[88] D. Nagar, S. Raghav, A. Bhardwaj, R. Kumar, P. L. Singh, and R. Sindhwani, "Machine learning: best way to sustain the supply chain in the era of industry 4.0," Materials Today: Proceedings, 2021.

[89] R. Sharma, S. S. Kamble, A. Gunasekaran, V. Kumar, and A. Kumar, "A systematic literature review on machine learning applications for sustainable agriculture supply chain performance," Computers and Operations Research, vol. 119, Article ID 104926, 2020.

[90] B. T. Hazen, I. Russo, I. Confente, and D. Pellathy, "Supply chain management for circular economy: conceptual framework and research agenda," The International Journal of Logistics Management, vol. 32, no. 2, pp. 510-537, 2021.

[91] H. Wilts, B. R. Garcia, R. G. Garlito, L. S. Gómez, and E. G. Prieto, "Artificial intelligence in the sorting of municipal waste as an enabler of the circular economy," Resources, vol. 10 , no. 4 , p. $28,2021$.

[92] D. D. Cremer, What Does Building a Fair AI Really Entail?, Harvard Business Review, Maasachussets, MA, USA, 2020, https://hbr.org/2020/09/what-does-building-a-fair-ai-reallyentail. 